\title{
Ein »neuer« Name aus der römischen Provinz Germania inferior Zur Inschrift AE 2006, 869 aus Erftstadt (Rhein-Erft-Kreis)
}

\author{
Andreas Kakoschke
}

\begin{abstract}
Zusammenfassung: In einer römerzeitlichen Steininschrift aus Erftstadt muss der Name Gaspenasianius (und nicht Gaspena Sianius) gelesen werden. Das Gentilnomen lässt sich auf den germanischen Namen *Gaspenasus zurückführen.
\end{abstract}

Abstract: In a stone inscription from Erftstadt dating to the Roman period, one should read the name Gaspenasianius (derived from the German *Gaspenasus), instead of Gaspena Sianius, as we find in an older transcription.

Im Tal des Rotbaches zwischen Mühlheim-Wichterich und Erftstadt-Niederberg fand sich vor einigen Jahren ein schlanker Inschriftenstein $(\mathrm{H} 70,2 \mathrm{~cm}-$ B 25,5 cm - T $22,4 \mathrm{~cm}$ ) aus Drachenfelstrachyt. Der beschädigte Stein, der vom Gelände einer benachbarten villa rustica stammt, wurde 2006 vom Rheinischen Landesmuseum Bonn erworben (Inv.-Nr. E 2006/75). In einem kleinen Artikel haben GERHARD BAUCHHENß und Petra TutLIES den Weihestein ausführlich vorgestellt. ${ }^{1}$ Den Text des Steines, welcher sich in doppelter (fast identischer) Ausführung auf der Vorder- (A) und der Rückseite (B) des Sockels befindet, geben die Autoren dabei wie folgt wieder:

A1

EIVS[ $[$ a. $3-4] C V R$
C(aius) Gaspna
Sianus
[C]assius
v(otum) s(olvit) l(ibens) m(erito).

B1 C(aius)•Gaspen-

$a \bullet$ Siani-

$u^{\bullet}$ Cassi-

$u s \bullet v($ otum $) \bullet s($ olvit $) \bullet$

B5 l(ibens) $m($ erito $)$.

Bis auf die Zeile A1, die keine sinnvolle Ergänzung zulässt, sind die Texte einwandfrei zu lesen. BAUCHHENß und TUTLIES gehen überzeugenderweise davon aus, dass der Dedikant zunächst die Inschrift A in Auftrag gab. Jedoch schlug der Steinmetz die Inschrift zum Leidwesen des Auftraggebers fehlerhaft ein. Offenbar hat dann ein zweiter Steinmetz (mit weniger akkuraten Buchstaben, einer anderen Laufweite der Schrift und schief gesetzten Zeilen) auf der Rückseite des Steins eine korrigierte Fassung des Textes mit Worttrennern (B) angebracht. Lautete der Name des Dedikanten in der älteren Inschrift noch C(aius) Gaspna Sianus Cassius, so wurde er jetzt als C(aius) Gaspena Sianius Cassius eingeschlagen. Der nicht besonders qualifizierte Steinmetz ergänzte also ein I in SIANVS und ein E in GASPNA, das er (vielleicht erst nachträglich) mit dem $\mathrm{P}$ ligierte. ${ }^{2}$ Laut BAUCHHENß und TutLIES besaß der Dedi-

\footnotetext{
${ }^{1}$ BAuchHENß/TutLies 2006, 134-137. Abb.131-133 (Photos). - Für Hinweise (wahrscheinlich für Hinweise zur Lesung und Interpretation der Inschrift) bedanken sich die Autoren ausdrücklich bei H. GALSTERER (Köln), der also allem Anschein nach die Ansichten der Autoren hinsichtlich der Lesung teilt. - Zur Inschrift s. ferner BAUCHHENß 2010, 522-523 (mit Photo).

${ }^{2} \mathrm{Zu}$ Steinmetzfehlern und nachträglichen Korrekturen in Inschriftentexten s. demnächst ausführlich KAKOSCHKE 2013. - Auf die Tatsache, dass in der älteren Inschrift A zwei Buchstaben im Namen des Dedikanten vergessen wurden, deutet auch die Zeilenaufteilung hin. Nur die Zeile A3 ist nicht ganz gefüllt. Am Zeilenende bietet sich noch Platz für zwei Buchstaben, für die im vorangegangenen Text vergessenen Buchstaben E und (das schmale) I. - BAuchHenß/TutLIES 2006, 136 vermerken zu recht, dass die Fehler des ersten Handwerkers noch nachträglich „mit einem dünnen Stucküberzug und richtiger Schreibung mit Farbe“ hätte behoben werden können. Allerdings
} 
kant damit zwei Gentilnomina, GASPENA und SIANIVS, die beide bisher unbekannt sind. ${ }^{3}$ Diese Interpretation ist m.E. nicht ganz zutreffend. Der Mann führte sicherlich eher die gängigen drei Namen (tria nomina) und hieß C(aius) Gaspenasianius Cassius. ${ }^{4}$ Der Worttrenner zwischen GASPENA und SIANIVS (Zeile B2) wurde dabei möglicherweise fehlerhaft gesetzt. Vielleicht ließ sich der Steinmetz durch die erste Fassung der Inschrift irritieren, in der GASPNA und SIANVS aufgrund des Zeilensprungs getrennt erscheinen. Es lässt sich jedoch nicht ausschließen, dass das lange Gentilnomen bewusst durch einen Worttrenner unterbrochen wurde. Verwiesen sei in diesem Zusammenhang auf eine Weihinschrift aus Morken-Harff (Rhein-Erft-Kr.), ca. $30 \mathrm{~km}$ nordwestlich von Köln, mit einer recht eigenwilligen silbentrennenden Inter-

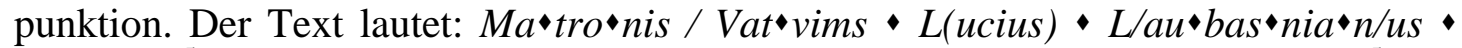

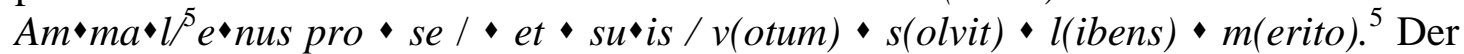
Name des Dedikanten, L. Laubasnian(i?)us Ammalenus, wurde hier (wahrscheinlich aus ästhetischen Gründen) gleich durch mehrere Worttrenner unterbrochen. Ferner kann eine weitere Weihinschrift aus dem näheren Raum, aus Nettersheim-Zingsheim (Kr. Euskirchen), angeführt werden. Das germanische Gentilnomen des Dedikanten, (C)hvaiionius (in dem das $\mathrm{CH}$ durch die rechte Längs- und die mittlere Querhaste eines $\mathrm{H}$ wiedergegeben wird) wurde als (C)HVAI•IONIVS in den Stein geschlagen. ${ }^{6}$ Da sich aus den zwei germanischen Provinzen weitere Belege für entsprechende Schreibungen mit Wortrennern innerhalb eines Namens erbringen lassen, ${ }^{7}$ wird man auch GASPENA•SIANIVS sicher ohne Bedenken als ein Gentiliz ansehen können.

Zwar erscheint das Pseudogentiliz Gaspenasianius, (natürlich) ein Hapax legomenon, auf den ersten Blick recht ungewöhnlich. Der Zungenbrecher ist jedoch nicht ungewöhnlicher als das in Xanten/CUT bezeugte „hinterwäldlerische“ Pseudogentiliz Verecundianius (mit der Basis Verecundus), ${ }^{8}$ das in Köln/CCAA nachgewiesene Simplicianius (mit der Basis Simplex) ${ }^{9}$ oder die aus Eschweiler-Fronhoven (Kr. Aachen) bekannte einheimische Bildung Negalatianius. ${ }^{10}$

Laut BAUCHHENß und TUTLIES liegen für Gaspena und Sianius keine verwandten Nachweise vor. ${ }^{11}$ Jedoch muss für Gaspenasianius auf das in Neuss/Novaesium belegte (Hapax legomenon) Gastinasus verwiesen werden. Der Name wird durch eine

\footnotetext{
wäre eine Korrektur mit Hilfe von Ligaturen viel einfacher (und gängiger) gewesen. So hätte der erste Steinmetz (wie sein Nachfolger) das vergessene E mit dem P verbinden können. Und durch eine Verlängerung der rechten Längshaste des N in SIANVS wäre man relativ leicht zu SIANIVS gelangt. Offenbar wurde dem Steinmetz eine Chance zu Korrekturen jedoch nicht mehr gewährt.

${ }^{3}$ Der vorgestellten Lesung entsprechend fand die Inschrift Aufnahme in die L'annee épigraphique (AE 2006, 869) und in die EDH (AE 2006, 00869). - Zu den zwei Namen s. ferner KAKOsChKE 2008, 491. Nr.522a und 492. Nr.1179a (Nachtrag).

${ }^{4}$ Zum Gentiliz Cassius in der Funktion als Cognomen bzw. nomen simplex s. KAKOsCHKE 2007, 218-219. Nr.718 (mit weiteren Hinweisen).

5 3. N. 205-206. Nr.233 = H. HoRN, in: RNRW 213. Abb.146 (Photo). - Eine entsprechende bemerkenswerte silbentrennende Interpunktion bietet in den germanischen Provinzen lediglich noch die Weihinschrift CIL XIII

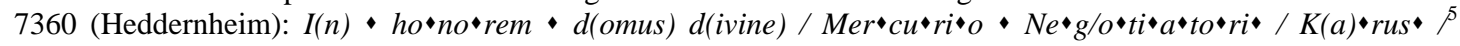
Tau^nen $s($ is $) \bullet / m o \bullet n i \bullet t u \bullet / d($ ei $) \bullet p($ osuit $) \bullet l($ ibens $) \bullet l($ aetus $) \bullet m($ erito $) \bullet$.

${ }^{6}$ RÜGER 1981, 288-289. Nr.2. Abb.2 (Photo) = AE 1977, 563a.

7 Verwiesen sei hier u.a. auf folgende Inschriften: CIL XIII 5493 (Dijon; AT•TI•CIL•LA), 5958 (Dijon; $\mathrm{MI} \bullet$ NVSILLA), 6145 (Eisenberg; GIA $\bullet$ MONIVS STATV $\bullet$ TVS), 6194 (Rehweiler; TASG•IL•LVS), 6279 (Nierstein; RO•MVLVS), 6316 [Iffezheim; C•L(audius)], 6347 (Hockenheim; SENI•LIS), 6449 (Benningen; T•ERMINVS), 6769 (Mainz; SA•TVRNINVS), 6813 (Mainz; GENIAE•LINEA), 7004 (Mainz; QVI•ETIVS), 7863 (Pattern; VER•ANIVS), 8346 (Köln; T•I•TVS), 8513 [Köln-Bocklemünd; NEG•ALAETI(us)], 8716 (Nijmegen; $\mathrm{LI} \bullet \mathrm{CINI} \bullet \mathrm{VS}), 11816[\mathrm{Mainz} ; \mathrm{F} \bullet \mathrm{L}($ avius $)]$.

${ }^{8}$ Bauchienß 1979, 22-23. Nr.10. Taf.7 (Photo) = 2. N. 116. Nr.243. Zum Namen s. KaKoschke 2006, 425. Nr.1391.

${ }^{9}$ CIL XIII 8423. Zum Namen s. KAKOSChKe 2006, 373. Nr.1190.

${ }^{10}$ RÜGER 1983, 154-156. Nr.39. Abb.39 (Photo) = AE 1984, 693. Zum Namen s. KaKOSChKe 2006, 283. Nr.845.

${ }^{11}$ BAUCHHENB/TutLIES 2006, 136.
} 
(wohl aus Köln/CCAA stammende) Ubierin namens Louba Gastinasi f(ilia) bezeugt. ${ }^{12}$ Gaspenasianius geht somit wahrscheinlich auf eine germanische Basis *Gaspenasus zurück.

BAUCHHENß und TUTLIES datieren den Weihestein des Mannes, in dem wir mit Sicherheit einen Einheimischen erblicken dürfen, ganz allgemein in die „Blütezeit“ der Weihegaben aus Stein, in die Jahre 150-250/260. ${ }^{13}$ Das Pseudogentiliz mit seinem Hang zur Länge, das über die Formen Gaspenasus bzw. Gaspenasianus erreicht wurde, bestätigt diesen Datierungsansatz, da es die provinziale Namensmode der Zeit um 200 wiederspiegelt. ${ }^{14}$

\section{Sigeln}

2. N.

3. N.

$\mathrm{AE}$

CIL

CSIR

EDF

RNRW

BAUCHHENß 1979

BAUCHHENß 2010

BAUCHHENß/TUTLIES 2006
H. Nesselhauf, Neue Inschriften aus dem römischen Germanien und den angrenzenden Gebieten, Bericht der Römisch-Germanischen Kommission 27 (1937), 51-134.

H. Nesselhauf, H. LIEB, Dritter Nachtrag zu CIL. XIII. Inschriften aus den germanischen Provinzen und dem Treverergebiet, Bericht der Römisch-Germanischen Kommission 40 (1959), 120-229.

L'annee épigraphique. Paris 1888ff.

Corpus Inscriptionum Latinarum. Berlin 1863ff.

Corpus Signorum Imperii Romani. Corpus der Skulpturen der Römischen Welt. Bonn.

Epigraphische Datenbank Frankfurt, Leitung: $M$. CLAUSS (Johann Wolfgang Goethe-Universität Frankfurt am Main), Stand: 15.11.2013 (www.manfredclauss.de).

Die Römer in Nordrhein-Westfalen, hrsg. von H. G. HORN, Stuttgart 1987.

\section{Literaturverzeichnis}

G. BAUCHHENß, Bonn und Umgebung. Zivile Grabdenkmäler, CSIR Deutschland III.2. Germania inferior, Bonn.

G. BAUCHHENß, Römischer Weihestein aus ErftstadtNiederberg, in: Fundgeschichten - Archäologie in Nordrhein-Westfalen, hrsg. von TH. ОтTEN, Mainz, 522-523.

G. BAUChHENß/P. TutLIES, Warum zweimal? Ein Trachytsockel aus dem Rotbachtal, Archäologie in Deutschland 134-137.

\footnotetext{
${ }^{12}$ CIL XIII 8565 = KAKoschKe 2002, 59-60. Nr.1.30. - Zum Namen Gastinasus s. SCHERER 1955, 206-207 (ubisch: „der die Gäste rettet“), Reichert 1987/1990, I. 309, KAKOSCHKE 2007, 377. Nr.1391 (mit weiteren Hinweisen).

${ }^{13}$ BAUCHHEN//TUTLIES 2006, 137.

${ }^{14}$ Eine Hilfestellung bei der Datierung anhand des Namens bietet Solin 1977, 103-146.
} 
KAKOSCHKE 2002

KAKOSCHKE 2006

KAKOSCHKE 2007

KAKOSCHKE 2008

KAKOSCHKE 2013

REICHERT 1987/1990

RÜGER 1981

RÜGER 1983

SCHERER 1955

SOLIN 1977
A. KAKOSCHKE, Ortsfremde in den römischen Provinzen Germania inferior und Germania superior. Eine Untersuchung zur Mobilität in den germanischen Provinzen anhand der Inschriften des 1. bis 3. Jahrhunderts n. Chr., Osnabrücker Forschungen zu Altertum und Antike-Rezeption 5, Möhnesee.

A. KAKoschKe, Die Personennamen in den zwei germanischen Provinzen. Ein Katalog. Bd.1: Gentilnomina ABILIUS-VOLUSIUS. Rahden/Westf.

A. KaKoschKe, Die Personennamen in den zwei germanischen Provinzen. Ein Katalog. Bd.2,1: Cognomina ABAIUS-LYSIAS. Rahden/Westf.

A. KAKOSCHKE, Die Personennamen in den zwei germanischen Provinzen. Ein Katalog. Bd.2,2: Cognomina MACCAUS-ZYASCELIS, Rahden/Westf.

A. KAKOSCHKE, Hapax - Steinmetzirrtum - Überlieferungsfehler - Fehllesung - Fälschung. $\mathrm{Zu}$ einigen auffälligen Personennamen aus den zwei germanischen Provinzen, Stuttgart (im Druck).

H. REICHERT, Lexikon der altgermanischen Namen, I. Text, II. Register, Österreichische Akademie der Wissenschaften. Schriftenreihe der Kommission für Altgermanistik. Thesaurus Palaeogermanicus 1, Wien.

CH. B. RÜGER, Inschriftenfunde der Jahre 1975-1979 aus dem Rheinland, Epigraphische Studien 12, 287-308.

CH. B. RÜGER, Römische Inschriftenfunde aus dem Rheinland 1978-1982, Epigraphische Studien 13, 111166.

A. SCHERER, Die keltisch-germanischen Namengleichungen, in: Corolla Linguistica. Festschrift. F. SOMMER zum 80. Geburtstag am 4.5.1955, hrsg. von H. KRAHE, Wiesbaden, 199-210.

H. SolIN, Die innere Chronologie des römischen Cognomens, in: Actes du Colloque International sur l'Onomastique Latine organisé à Paris du 13 au 15 octobre 1975 par H.-G. Pflaum et N. DuVAL, Paris, 103-146.

\section{Kontakt zum Autor:}

Dr. Andreas Kakoschke

Schamelweg 4

D-49191 Belm

Email: andreaskakoschke@ hotmail.de 